\section{Development of artificial neural network-based model for prediction of temperature field in host rock of a geological disposal facility for radioactive waste}

\author{
Vrinda Brajesh Gupta*, T. K. Pal and \\ R. K. Bajpai
}

Bhabha Atomic Research Centre, Mumbai 400 085, India

Calculation of temperature field in a deep geological repository (DGR) after emplacement of a large number of heat emitting vitrified radioactive canisters is important and requires large computational time and hence in this study an effort has been made towards development of artificial neural network (ANN) based model that can predict the temperature quickly. The datasets required to train the ANN model were generated using an in-house developed GUI tool for simulating heat diffusion process. Various numerical studies were conducted with different configurations of the ANN model and different datasets of size 50, $100,150,200$, to optimize the number of input data required to train the model. The results in the form of temperature values predicted by the trained ANN model have been compared with those for the same problem calculated using analytical and finite difference based methods. The trained ANN model can predict temperature values with less than $0.001 \%$ error.

Keywords: Artificial neural network, geological repository, host rock, radioactive waste, temperature field.

WORLD over spent nuclear fuels and vitrified high-level heat-emitting long-lived radioactive waste are under consideration for disposal in geological disposal facilities, popularly known as deep geological repositories (DGRs) ${ }^{1}$. These facilities can provide isolation and confinement to such waste over tens of thousands of years till their radioactivity decays down to safe level given by regulatory authorities ${ }^{2,3}$.

It is expected and has also been verified by various numerical and in situ laboratory-scale studies carried out in India ${ }^{4-6}$ as well as in other countries ${ }^{7,8}$ that the heat generated by the spent nuclear fuel/vitrified high-level waste disposed in a DGR will increase the temperature in surrounding clay-based buffer, backfill and host rocks. Though countries such as USA, Sweden and Finland, where DGRs under consideration are for spent fuel disposal, consider temperature more than $200^{\circ} \mathrm{C}$ in the host rock due higher heat flux from their waste-loaded canisters, in countries, such as India and France, where spent fuel is reprocessed to recover important isotopes having

\footnotetext{
*For correspondence. (e-mail: vrindageda@gmail.com)
}

societal applications, the resultant high-level waste after vitrification and pre-disposal storage of about 40 years has significant lower heat flux $(\sim 500 \mathrm{~W} /$ vitrified HLW canister). Hence disposal of such canisters in the DGR at appropriate spacing between canisters and various disposal tunnels would not produce temperature above $100^{\circ} \mathrm{C}$ in any part of such waste disposal facilities at any point of time over extended periods ${ }^{2,5}$. The factors that affect the temperature field in these facilities are heat flux from the canister at the time of disposal, heat decay, thermal properties of the rock and clay, orientation of the waste-loaded canisters, initial undisturbed rock temperature at the DGR depth and the DGR layout (Figure 1).

Thermal conductivity, specific heat capacity and density of rock have a direct impact on temperature. The higher the thermal conductivity, the more quickly heat is conducted through the rock and away from the canisters. Temperature is inversely proportional to density and specific heat capacity, whereas it is directly proportional to thermal conductivity.

The general three-dimensional heat diffusion equation in the geological medium can be written as

$$
\begin{aligned}
& \frac{\partial T(x, y, z)}{\partial t} \\
& =D\left(\frac{\partial^{2} T(x, y, z)}{\partial x^{2}}+\frac{\partial^{2} T(x, y, z)}{\partial y^{2}}+\frac{\partial^{2} T(x, y, z)}{\partial z^{2}}\right),
\end{aligned}
$$

where $D=k / \rho C_{\mathrm{v}}$ is the thermal diffusion coefficient.

The finite difference equation for $3 \mathrm{D}$ heat diffusion is

$$
\begin{aligned}
& T(x, y, z, t+\Delta t)=T(x, y, z, t)[1-2 a-2 b-2 c] \\
& \quad+a[T(x+\Delta x, y, z, t)+T(x-\Delta x, y, z, t)] \\
& \quad+b[T(x, y+\Delta y, z, t)+T(x, y-\Delta y, z, t)] \\
& \quad+c[T(x, y, z+\Delta z, t)+T(x, y,-\Delta z, t)]
\end{aligned}
$$

where

$$
a=\frac{D \Delta t}{\Delta x^{2}}, b=\frac{D \Delta t}{\Delta y^{2}}, c=\frac{D \Delta t}{\Delta z^{2}},
$$

with stability condition

$$
\Delta t \leq \frac{\Delta x^{2} \Delta y^{2} \Delta z^{2}}{2 D\left(\Delta x^{2} \Delta y^{2}+\Delta x^{2} \Delta z^{2}+\Delta y^{2} \Delta z^{2}\right)} .
$$

In India, granite rocks due to their vast occurrence throughout the country and adequate thermo, mechanical and hydrological characteristics, have been considered as target host rocks for developing DGR. For the present 
study, medium grained-granite host rock belonging to Jalore granite of northern Rajasthan and clay samples from Akli formation of Bermer district in northern Rajasthan were tested in the laboratory for their thermal parameters ${ }^{9}$. Table 1 shows the values of parameters of the disposal environment. Figure 1 shows the actual layout of the waste disposal pit. Figure 2 is a diagrammatic representation of a typical waste canister along with various components like buffer, rocks and their dimensions in the disposal environment. Table 2 shows the experimental range of thermal conductivity, specific heat capacity and density of rocks. Table 3 shows the experimental values of thermal conductivity, specific heat capacity and density of clay.

The ANN model is a computational model whose working is analogous to the biological neurons. ANN consists of a large number of simple processing elements or neurons which are interconnected by means of directed communication links having some associated weight and operate in parallel ${ }^{10}$.

Single-layer network is the simplest form in which the input layer directly projects into the output layer of the neurons. Multilayer network on the other hand, consists of one or more hidden layers between input and output layers, which are able to extract higher-order statics and thus more useful ${ }^{11}$.

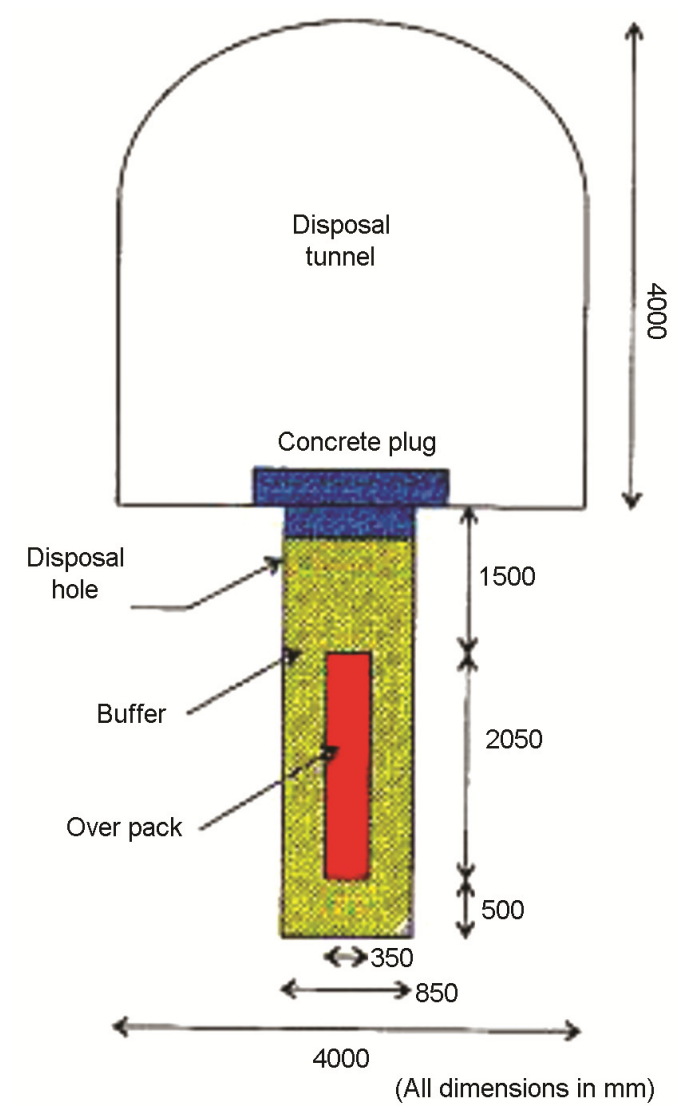

Figure 1. Actual layout of a waste disposal pit.
Multi-layer network or perceptron is trained with backpropagation algorithm, which is a type of supervised learning algorithm. Training and learning procedure starts with forward propagation in which inputs are multiplied by a random set of weight values (generally between 0.5 and -0.5). This weighted sum of inputs forms the net input to neurons. Net input can be written as

$$
a_{i}=\sum_{j=1}^{n} W_{i j} X_{j}+C_{i} \text {, }
$$

where $X_{j}, j=1, \ldots, n$, is output from the $j$ th neuron of the previous layer, $W_{i j}$ are weights connecting neuron $j$ to neuron $i$ and $C_{i}$ is a threshold for neuron $i$. Each neuron acts as an activation function to produce the output.

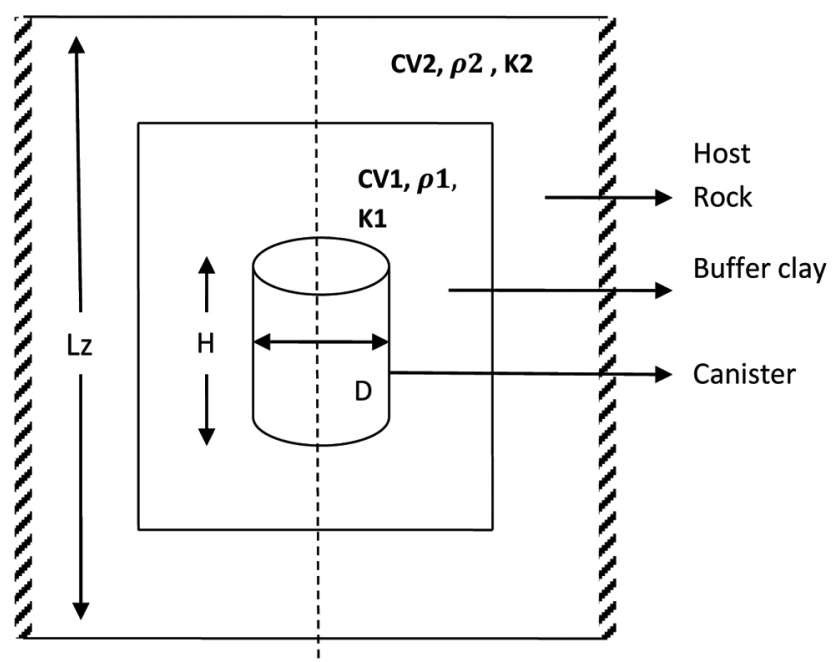

Figure 2. Diagrammatic representation of the waste disposal environment.

Table 1. Model parameters and their values

\begin{tabular}{lc}
\hline Parameters & Values \\
\hline Domain length along $z$ direction & $10 \mathrm{~m}$ \\
Diameter of canister $(D)$ & $0.36 \mathrm{~m}$ \\
Height of canister $(H)$ & $2 \mathrm{~m}$ \\
Initial undisturbed rock temperature $\left(T_{0}\right)$ & $40^{\circ} \mathrm{C}$ \\
Initial power of canister $\left(P_{0}\right)$ & $500 \mathrm{~W} / \mathrm{over}^{\mathrm{pack}}$ \\
Decay constant $(\lambda)$ & $7.32496 \times 10^{-10} \mathrm{sec}^{-1}$ \\
Monitoring time & 10.0 days \\
Distance of point from the canister where & $2.0 \mathrm{~m}$ \\
$\quad$ temperature is being calculated & \\
\hline
\end{tabular}

Table 2. Physical parameters of granite rock and their range

\begin{tabular}{lc}
\hline Parameters & Range \\
\hline Thermal conductivity $\left(\mathrm{K}_{2}\right)$ & $3-4.5 \mathrm{~W} / \mathrm{m} / \mathrm{K}$ \\
Material density $\left(\rho_{2}\right)$ & $2000-3000 \mathrm{~kg} / \mathrm{m}^{3}$ \\
Specific heat capacity $\left(C_{\mathrm{v} 2}\right)$ & $900-1100 \mathrm{~J} / \mathrm{kg} / \mathrm{K}$ \\
\hline
\end{tabular}

CURRENT SCIENCE, VOL. 118, NO. 3, 10 FEBRUARY 2020 


\begin{tabular}{lc}
\hline \multicolumn{1}{c}{ Table 3. } & Physical parameters of clay \\
\hline Parameters & Range \\
\hline Thermal conductivity $\left(K_{1}\right)$ & $0.78 \mathrm{~W} / \mathrm{m} / \mathrm{K}$ \\
Material density $\left(\rho_{1}\right)$ & $590.0 \mathrm{~kg} / \mathrm{m}^{3}$ \\
Specific heat capacity $\left(C_{\mathrm{v} 1}\right)$ & $1600.0 \mathrm{~J} / \mathrm{kg} / \mathrm{K}$ \\
\hline
\end{tabular}

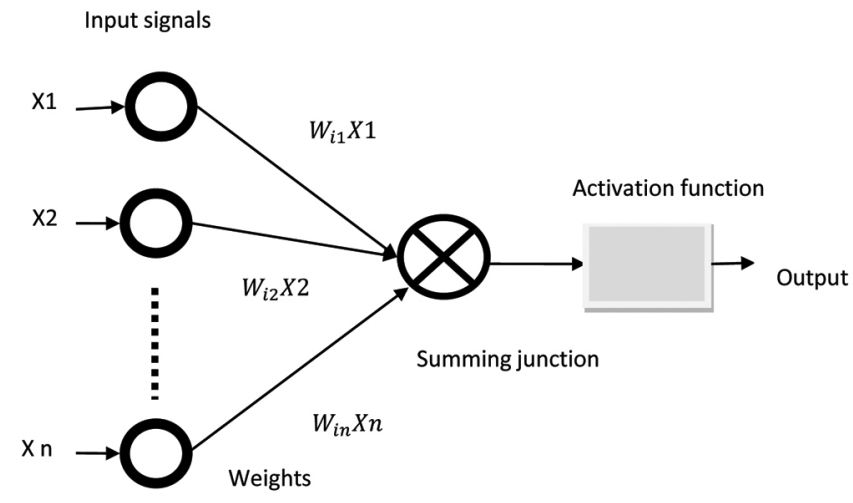

Figure 3. Mathematical model of artificial neural network.

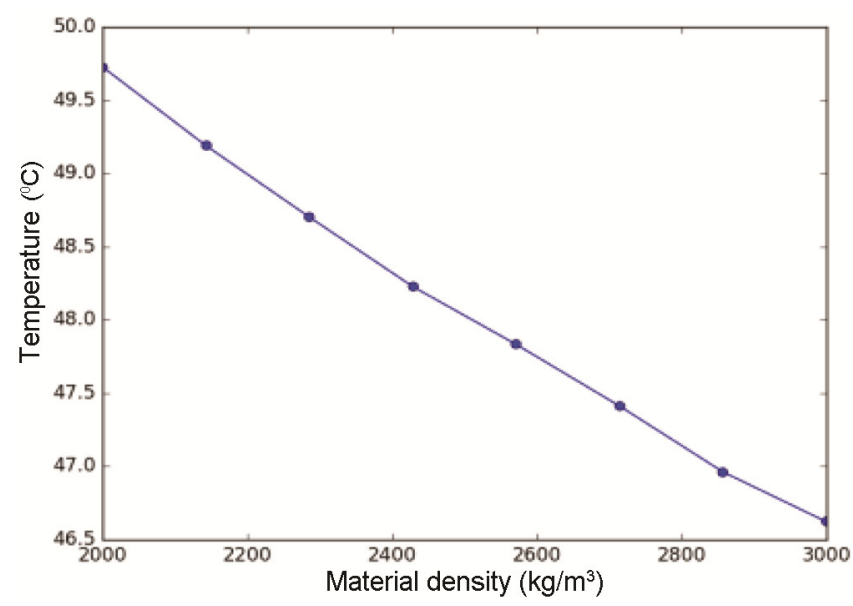

Figure 4. Variation of temperature with material density.

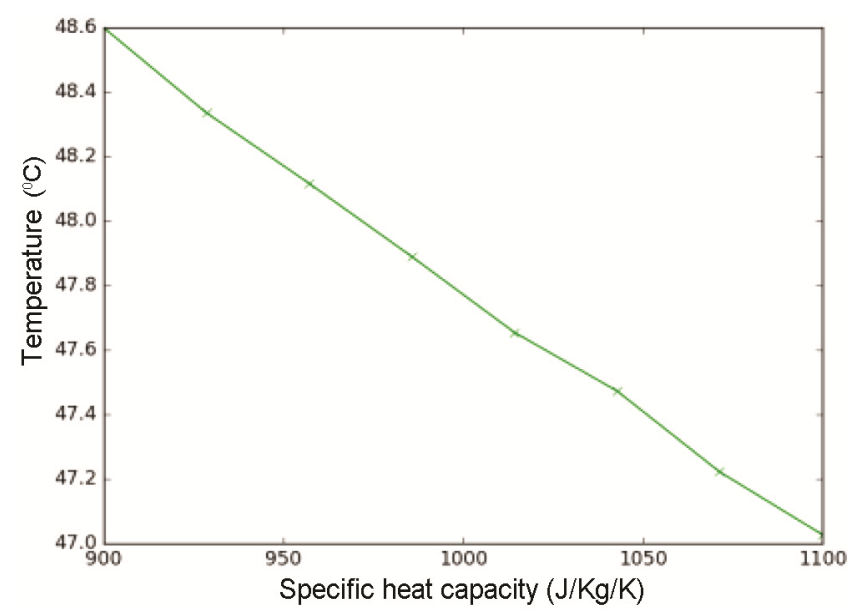

Figure 5. Variation of temperature with specific heat capacity.

$$
b_{i}=f\left(a_{i}\right)=f\left(\sum_{j=1}^{n} W_{i j} X_{j}+C_{i}\right)
$$

where $f($.$) is the activation function.$

Generally sigmoid function is used as an activation function to normalize the inputs between +1 and 0 . The sigmoid function can be defined as

$$
Y=\frac{1}{1+\mathrm{e}^{-\left(\sum\left(W_{i} X_{i}\right)\right)}} .
$$

Error is calculated and then in back propagation, error signals are back propagated through the network layer by layer to obtain the desired output (Figure 3$)^{12,13}$.

Table 4. Architecture of artificial neural network model

\begin{tabular}{lc}
\hline Parameters & Values \\
\hline No. of input neurons & 3 \\
No. of output neurons & 1 \\
Targeted error & 0.001 \\
Learning rate & 0.7 \\
\hline
\end{tabular}

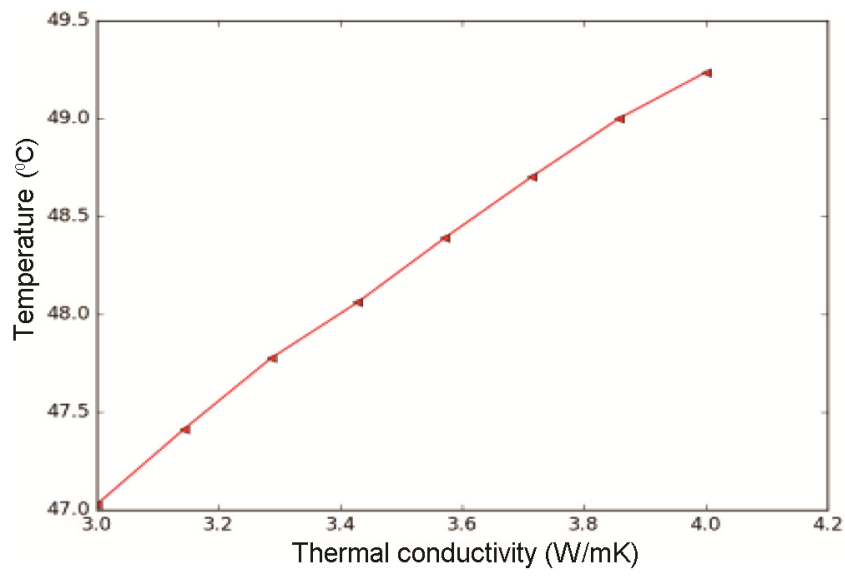

Figure 6. Variation of temperature with thermal conductivity.

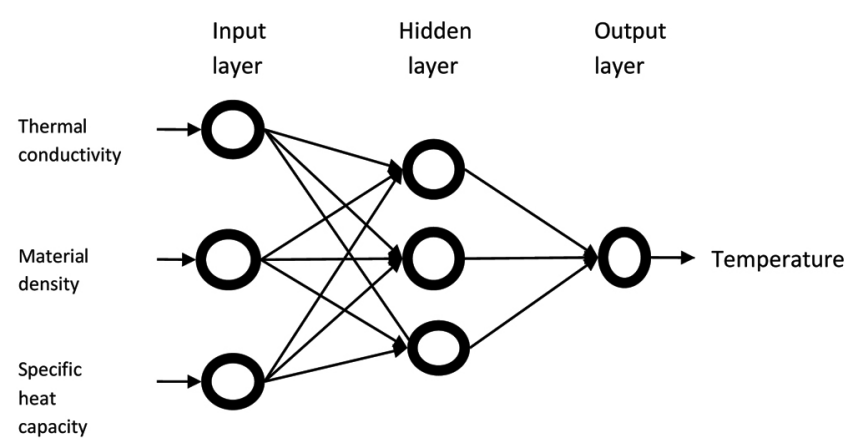

Figure 7. Multilayer feed-forward network with one hidden layer, and input and output parameters. 
Table 5. Comparison of absolute percentage average error for different datasets

\begin{tabular}{lccccc}
\hline & & \multicolumn{4}{c}{ Absolute \% average error } \\
$\begin{array}{l}\text { Number of } \\
\text { hidden layers }\end{array}$ & $\begin{array}{c}\text { Number of } \\
\text { neurons }\end{array}$ & 50 & 100 & 150 & 200 \\
\cline { 3 - 6 } 1 & 1 & 0.458896 & 0.216559 & 0.097051 & 0.062898 \\
1 & 2 & 0.441054 & 0.189000 & 0.071197 & 0.040179 \\
1 & 3 & 0.280584 & 0.017843 & 0.069425 & 0.019545 \\
2 & 1 & 0.340782 & 0.180689 & 0.062609 & 0.032154 \\
2 & 2 & 0.334658 & 0.098333 & 0.051063 & 0.003410 \\
2 & 3 & 0.329496 & 0.076749 & 0.022512 & 0.002090 \\
3 & 1 & 0.357712 & 0.202957 & 0.038050 & 0.038440 \\
3 & 2 & 0.328163 & 0.108973 & 0.022297 & 0.003813 \\
3 & 3 & 0.269398 & 0.049166 & 0.005446 & 0.000677 \\
\hline
\end{tabular}

Table 6. Variation of error with the number of datasets

\begin{tabular}{rc}
\hline Number of datasets & Absolute \% average error \\
\hline 50 & 0.280584 \\
100 & 0.017843 \\
150 & 0.069425 \\
200 & 0.019545 \\
\hline
\end{tabular}

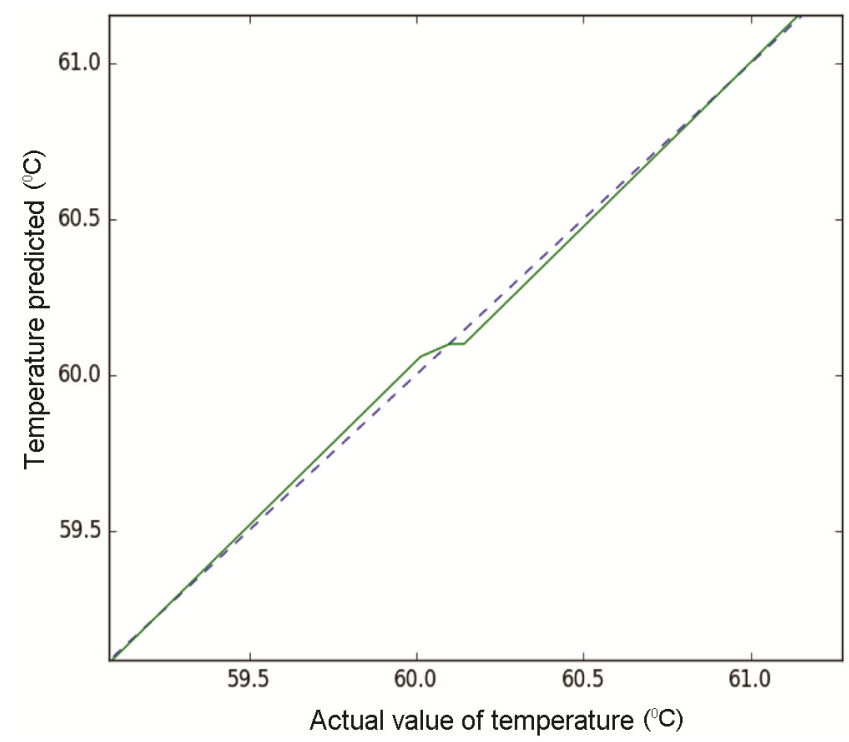

Figure 8. Graph showing actual value of temperature versus predicted temperature.

A numerical study was carried out to determine how temperature at a given spatio-temporal location in the DGR varies with various thermal properties of the rocks such as thermal conductivity, specific heat and density. Considering that these thermal properties of the rocks are uniformly distributed, random samples of these parameters are generated from the lower and upper limits of input values. Table 2 shows the range of input parameter values used for random sampling. Using these input parameter values, temperatures at a given spatio-temporal location are calculated using in-house developed GUI tool. Since we have three input parameters, three different studies were carried out where one input parameter was taken as the random variable in each study, while the other two parameters were considered as constant. Figures 4-6 show result of temperature variation with variation in material density, specific heat and thermal conductivity respectively. The graphical results show expected variation in temperature with these parameters. At this stage it is possible to generate a response surface of temperature by varying all the three parameters simultaneously. However, this kind of study would not help us to establish a functional relationship between temperature and the input parameters. Basically, this task of establishing the unknown relationship between temperature and the three input parameters is done using the ANN model developed in the present study. Considering thermal conductivity, specific heat capacity and density of rocks as known parameters, temperature being unknown, an ANN model with three input parameters, i.e. thermal conductivity, specific heat capacity and density of rocks, and one output parameter, i.e. temperature is constructed (Figure 7). The targeted rate was set to $0.001 \%$ and the learning rate to 0.7 . Table 4 shows the architecture of the ANN network.

The datasets required for training and testing of the constructed ANN model and for verification of the model output are generated using in-house developed GUI tool. Once training is completed, testing and prediction of the model are done. Error between actual temperature and predicted temperature is calculated. Here the actual temperature refers to the temperature calculated using the GUI tool and therefore, numerical model uncertainties are expected to be propagated though the ANN model.

The ANN model was run with different number of hidden layers, neurons and datasets, i.e. 50, 100, 150 and 200 (Table 5). In each dataset 10 random sample values were used for prediction and the rest used for training and testing. The dataset for training, testing and prediction of the ANN model was generated by solving 3D heat diffusion equation using finite difference method (eq. (2)). 
RESEARCH COMMUNICATIONS

Table 7. Analysis of simulated and predicted values of temperature

\begin{tabular}{lccccc}
\hline $\begin{array}{l}\text { Thermal } \\
\text { conductivity }\end{array}$ & $\begin{array}{c}\text { Material } \\
\text { density }\end{array}$ & $\begin{array}{c}\text { Specific } \\
\text { heat capacity }\end{array}$ & $\begin{array}{c}\text { Actual } \\
\text { temperature }\end{array}$ & $\begin{array}{c}\text { Predicted } \\
\text { temperature }\end{array}$ & $\begin{array}{c}\text { Absolute \% } \\
\text { average error }\end{array}$ \\
\hline 3.43564 & 2760.0 & 990.0 & 57.6548 & 57.682305 & 0.047706 \\
4.11811 & 2330.0 & 1020.0 & 60.0586 & 60.096961 & 0.063884 \\
3.16780 & 2570.0 & 940.0 & 57.9472 & 58.001588 & 0.093858 \\
4.17074 & 2760.0 & 1090.0 & 58.3893 & 58.396818 & 0.012876 \\
4.19418 & 2780.0 & 1000.0 & 59.0470 & 59.054747 & 0.013120 \\
4.40849 & 2760.0 & 920.0 & 60.0991 & 60.018129 & 0.013456 \\
4.43258 & 2330.0 & 930.0 & 61.2529 & 61.248358 & 0.007414 \\
3.36730 & 2600.0 & 950.0 & 58.2818 & 58.308116 & 0.045154 \\
3.40642 & 2940.0 & 920.0 & 57.6548 & 57.635079 & 0.034203 \\
4.41311 & 2480.0 & 1020.0 & 60.0987 & 60.143737 & 0.074939 \\
\hline
\end{tabular}

Absolute \% average error $=0.017$.

Table 6 shows variation of error with different number of datasets $(50,100,150$ and 200) used for training the model with one hidden layer and three neurons.

When the model is run with one hidden layer, three neurons and dataset of the 100, absolute percentage error was found to be 0.017843 which is small and acceptable. Table 7 shows a comparison between simulated and predicted temperatures along with associated absolute percentage error and values of parameters (thermal conductivity, specific heat capacity, density of rocks) with this configuration. Figure 8 shows that the plot between actual value of temperature and predicted temperature is almost equal.

The ANN model is trained to predict temperature within the host rocks in DGR at a particular sampling point and monitoring time. The developed ANN model can be used with different types of rocks having different values of thermal conductivity, specific heat capacity and material density. Absolute percentage error for 50 datasets, i.e. 0.458896 attains a value of 0.062898 as number of datasets increases to 200. Also with increase in the number of hidden layers and number of neurons in 50 datasets, absolute percentage error falls from 0.458896 to 0.269398 . For this case study, single hidden layer with three neurons run with 100 datasets is sufficient to get precise results. The percentage error was found out to be around $0.01 \%$. The percentage error is small and within acceptable limits, so the ANN model is reliable. Moreover, back propagation algorithm is simple to implement and provides effective solutions to large problems.

1. Rempe, N. T., Permanent underground repositories for radioactive waste. Prog. Nucl. Energy, 2007, 49, 365-374.

2. Narayan, P. K. and Bajpai, R. K., Deep geological repositories for vitrified high level long lived wastes. IANCAS Bull., 2007, VI, 224-237.

3. Bajpai, R. K., Recent advances in the geological disposal of nuclear-wastes worldwide and Indian scenario. J. Geol. Soc. India, 2004, 63(3), 354-356.

4. Maheshwar, S., Verma, A. K., Singh, T. N. and Bajpai, R. K., A numerical study of the thermal interferences between two nuclear waste canisters in a granite hosted repository. Int. J. Earth Sci. Eng., 2015, 8(3), 1089-1098.

5. Mathur, R. K., Narayan, P. K., Joshi, M. R. and Rakesh, R. R., In situ multi-heater thermomechanical experiments in Mysore Mines, Kolar gold fields. BARC report BARC/1998/I/015, Bhabha Atomic Research Centre, Mumbai, 1998.

6. Maheshwar, S., Verma, A. K., Singh, T. N. and Bajpai, R. K., Study of thermo-hydro-mechanical processes at a potential site of an Indian nuclear waste repository. J. Earth Syst. Sci., 2015, 124(8), 1693-1708.

7. Kwon, S., Cho, W. J. and Lee, J. O., An analysis of the thermal and mechanical behavior of engineered barriers in a high-level radioactive waste repository. Nucl. Eng. Technol., 2013, 45(1), $41-52$.

8. Lee, J. O., Choi, H. and Kim, G. Y., Numerical simulation studies on predicting the peak temperature in the buffer of an HLW repository. Int. J. Heat Mass Transfer, 2017, 115, 192-204.

9. Goel, R. K., Prasad, V. V. R., Swarup, A., Dwivedi, R. D., Mohnot, J. K., Soni, A. K. and Misra, D. D., Testing of rock samples and site-specific design of underground research laboratory. Technical Report, Central Mining Research Institute, Roorkee, GC/MT/R/1/2000-2001, pp. 1-64.

10. Ortiz-Rodríguez, J. M., del Rosario Martínez-Blanco, M., Viramontes, J. M. C. and Vega-Carrillo, H. R., Robust design of artificial neural networks methodology in neutron spectrometry. In Artificial Neural Networks-Architectures and Applications, InTech Open Access Publisher, Croatia, 2013.

11. Wai-Shing, C. T. and Siu-Yeung, C. D., Neural Networks and Computing: Learning Algorithms and Applications, Imperial College Press, London, 2007.

12. Haykin, S., Neural Networks: A Comprehensive Foundation, Prentice Hall PTR, Macmillan, New York, 1994.

13. Yadav, N., Yadav, A. and Kumar, M., An Introduction to Neural Network Methods for Differential Equations, The Netherlands, Springer, 2015.

Received 22 June 2019; revised accepted 2 October 2019

doi: $10.18520 / \mathrm{cs} / \mathrm{v} 118 / \mathrm{i} 3 / 439-443$ 